\title{
Ecologie des Leishmanioses dans le sud de la France
}

\author{
2. - Les réservoirs selvatiques* \\ Infestation spontanée du Renard (Vulpes vulpes L.) \\ par J.-A. RIOUX, J.-L. ALBARET, R. HOUIN, \\ J.-P. DEDET et G. LANOTTE \\ [Laboratoire d'Ecologie médicale. Faculté de Médecine, 34 -Montpellier \\ et Laboratoire de Zoologie (Vers). Muséum National d'Histoire Naturelle, \\ 57, rue Cuvier - F 75 - Paris-5 $\left.{ }^{\circ}\right]$
}

\begin{abstract}
Résumé
Poursuivant leur enquête sur les foyers leishmaniens du sud de la France, les auteurs étudient la réceptivité expérimentale et l'infestation spontanée des réservoirs selvatiques. Expérimentalement, les Rongeurs, et particulièrement les Gliridés, se montrent réceptifs aux trois types de Leishmanies, dermotropes et viscérotropes (humaine et canine) ; toutefois, aucune infestation naturelle n'est observée. Par contre, deux Renards, capturés aux limites du foyer cévenol, sont trouvés spontanément atteints. L'inoculation d'un broyat de ganglion parasité à deux autres Renards, provenant d'une région indemne, est suivie d'infestation.
\end{abstract}

\section{Summary}

Following their investigation about the focus of Leishmania in Southern France, the authors study the experimental receptivity and the spontaneous infection of the selvatic reservoirs. Experimentally, the rodents, and particularly the Gliridae, appear to be receptive to the three types of Leishmania, dermotropic and viscerotropic (human and canine); however no natural infection is observed. On the other hand two foxes captured on the limits of the Cevenol focus were found spontaneously infected. The inoculation of a crushed parasitized lymph gland to two foxes, coming from an uninfected region, is followed by an infection.

* Collaboration technique: A. Bres, A. Dumas et M. Maistre. 
Depuis les travaux des épidémiologistes russes (U. P. Vlasov, 1931 ; P. A. Petrishcheva, 1935 ; N. I. Latyshev, A. P. Kryukova et T. P. Povalishina, 1951), nous savons, qu'à l'image de nombreuses zoonoses, les leishmanioses évoluent à l'intérieur de territoires géographiquement limités ou « foyers naturels », dans lesquels l'affection perdure tant que n'intervient aucune modification bioclimatique ou édaphique. Le parasite se perpétue sur un ensemble de Vertébrés réceptifs, grâce à l'intervention d'un vecteur spécifique : le Phlébotome.

Avec P. C. Garnham (1965), il est à présent classique de distinguer des «foyers primaires» où le réservoir est constitué par le seul Vertébré sauvage (Carnivores ou Rongeurs) et des «foyers secondaires » dans lesquels un Vertébré domestique se substitue aux hôtes précédents.

De nombreux auteurs situent actuellement les foyers primaires de Leishmaniose viscérale sur les contreforts montagneux du Tadjikistan et de la Kirghizie, aux confins des grandes steppes de l'Asie Centrale. Dans ces foyers, la zoonose est entretenue par des Canidés sauvages (Canis lupus L., Canis aureus L., Vulpes vulpes L.) ; l'agent pathogène est transmis par des Phlébotomes de comportement foncièrement zoophile. L'intrusion de l'Homme dans un tel «complexe » est inhabituel et, partant, sa contamination exceptionnelle.

Dans les foyers secondaires, le Chien occupe la place du réservoir sauvage, soit que ce réservoir n'ait jamais existé, soit qu'il ait été peu à peu éliminé par la chasse, la culture ou l'élevage. La zoonose se développe alors au contact même de l'Homme dont la contamination est inéluctable dès l'instant où existe un vecteur à double potentialité trophique (anthropo-zoophile). Ainsi en est-il des foyers chinois, brésiliens et méditerranéens.

Toutefois, le cycle épidémiologique classique Chien-Phlébotome-Homme, caractéristique des foyers secondaires, n'exprime pas toujours l'intégralité des faits. Ainsi, dans certains pays, réservoirs domestiques et réservoirs sauvages coexistent, par exemple dans l'Etat de Ceara au Brésil, où le Renard des Savanes, Lycalopex vetulus (Lund, 1848) est atteint au même titre que le Chien (M. Deane et L. M. Deane, 1954). On comprend que de telles observations puissent remettre en question la structure même du «complexe pathogène » et, partant, les options générales de lutte: dans ce cas en effet, on est en droit de se demander si l'hôte sauvage se comporte effectivement comme un « réservoir habituel », donc susceptible d'entretenir l'affection sans le secours du Chien ou comme un « réservoir occasionnel », c'est-à-dire directement dépendant de l'endémie canine. Dans cette dernière éventualité, où le cycle selvatique n'a pas d'existence propre, l'éradication de la maladie peut être obtenue théoriquement par l'élimination sélective des Chiens atteints.

Dans le cadre de l'enquête épidémiologique que nous poursuivons depuis 1961 sur le foyer leishmanien des Cévennes méridionales, nous avons entrepris le dépistage des réservoirs sauvages hautement probables: Rongeurs et Carnivores. Comme il se doit, une étude de la sensibilité expérimentale a été menée parallèlement sur les mêmes espèces. 


\section{$1^{\circ}$ Sensibilité expérimentale.}

Au cours de cette étude, 567 animaux ont été inoculés à partir de Leptomonas de culture. Les trois types de souches autochtones, humaines (viscérale et cutanée) et canines ont été utilisées. Avant l'inoculation, chaque animal a fait l'objet d'une splénectomie partielle de manière à éliminer une éventuelle infestation spontanée. Les résultats globaux, résumés dans le tableau 1, montrent une nette sensibilité des Gliridés à l'ensemble des trois types de souches; les Muridés sont moins sensibles. Les Carnivores, en particulier les Canidés, se révèlent totalement résistants. Ce dernier résultat, en contradiction apparente avec les faits, sera discuté plus loin.

$2^{\circ}$ Infestation naturelle.

Cette enquête nous a amenés à examiner 1.130 animaux, répartis comme suit :

\section{REPTILES}

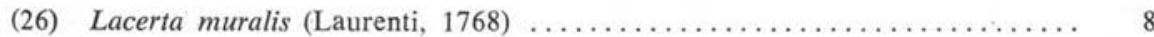

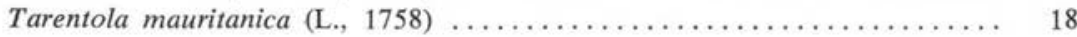

\section{CARnivores}

(224) Vulpes vulpes $(\mathrm{L} ., 1758) \ldots \ldots \ldots \ldots \ldots \ldots \ldots \ldots \ldots \ldots \ldots \ldots \ldots \ldots$

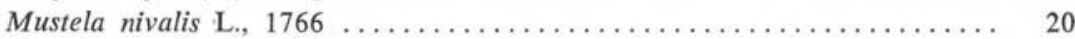

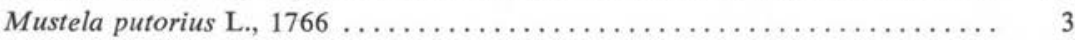

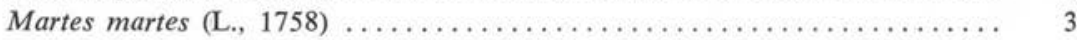

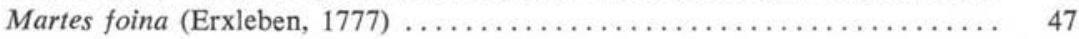

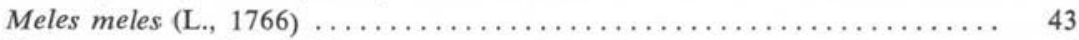

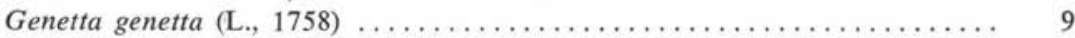

RONGEURS

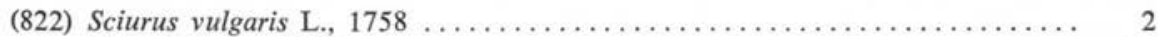

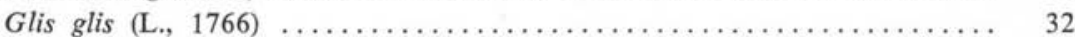

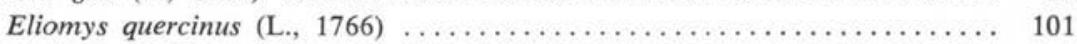

Clethrionomys glareolus (Schreber, 1780) .................... 1

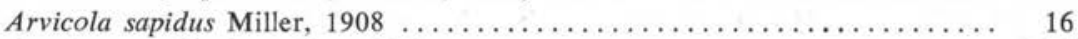

Apodemus sylvaticus (L., 1758) ........................... 505

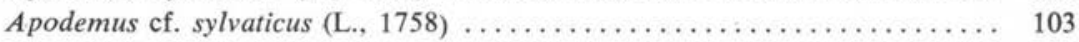

Apodemus flavicollis (Melchior, 1834) ..................... 4

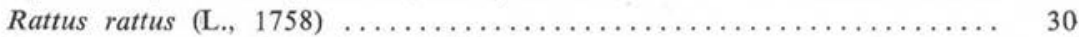

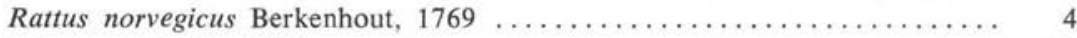

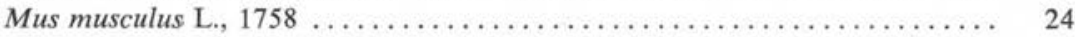

\section{INSECTIVORES}

(57) Erinaceus europaeus L., 1758 (s.l.) . . . . . . . . . . . . . . . . . 37

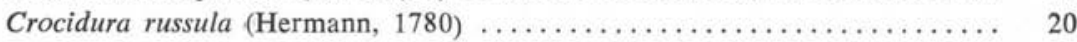

La plupart des animaux transportés vivants au laboratoire ont été sacrifiés dans les heures ou les jours suivant leur capture. La recherche des Leishmanies s'est effectuée 
sur frottis colorés et culture en milieu NNN (prélèvements de sang, foie, rate, moelle osseuse et poumon).

A l'occasion de cette prospection, un assez grand nombre de Protozoaires sanguicoles ou réticulotropes ont été mis en évidence, ainsi :

a) des Piroplasmidés chez 5 Blaireaux (Babesia missirolii Biocca et Coradetti, 1952) et chez 9 Hérissons (Piroplasma sp.);

b) des Sporozoaires coccidiomorphes du genre Hepatozoon chez Lacerta muralis, Vulpes vulpes (Hepatozoon canis James, 1905), et Apodemus sylvaticus;

c) des Trypanosomes chez:

4 Tarentola mauritanica ........... Trypanosoma cf. platydactili (Catouillard, 1901).

4 Rattus rattus ................. Trypanosoma lewisi (Kent, 1880).

13 Eliomys quercinus .............. Trypanosoma blanchardi Brumpt, 1905.

33 Meles meles ................. Trypanosoma pestanai Bettencourt et França, 1905.

1 Mustela nivalis ................. Trypanosoma sp.

d) des Leishmanies (Leishmania tarentolae) associées aux Trypanosomes dans le sang de deux Géckos.

Enfin, sur 99 Renards examinés, deux cas de leishmaniose viscérale ont pu être dépistés :

Le premier individu, capturé le 24 avril 1963 à Saint-Mathieu-de-Tréviers, au nord de Montpellier, ne présentait aucun signe clinique et seule la culture de la moelle osseuse mettait en évidence le parasite. Le second exemplaire, piégé dans la même localité, accusait un état de grande maigreur; de larges plaques de dépilation avec furfur s'étendaient sur le ventre et la face interne des cuisses. Les frottis de rate et de moelle osseuse, pratiqués le jour même de la capture, montraient de nombreuses Leishmanies. La culture de moelle était elle-même positive.

Dès lors, la découverte d'une infestation naturelle du Renard nous amenait à reconsidérer les résultats de nos inoculations expérimentales. Comme dans les tentatives identiques menées sur le Chien par C. Nicolle (1908), les échecs que nous avions enregistrés semblaient dus en grande partie à la perte du pouvoir pathogène des souches de culture : l'inoculation du Renard par la forme leishmanienne s'imposait.

Le 16 janvier 1967, deux Renards adultes, provenant d'un élevage des environs de Toulouse, recevaient par les voies sous-cutanée $(4 \mathrm{ml})$ et intra-veineuse $(12 \mathrm{ml})$ le broyat d'un ganglion de chien leishmanien ; 60.000 Leishmanies étaient ainsi inoculées à chaque animal. Un troisième sujet (2.961) servait de témoin.

Le 17 août 1968, à l'occasion d'une prise de sang, on constatait un état de maigreur anormal chez l'un des exemplaires inoculés (2.963). Ce dernier mourait en cours d'anesthésie. Les divers prélèvements de rate, de foie et moelle fourmillaient de Leishmanies. Les primo-cultures de ces mêmes organes étaient positives ainsi que 


\begin{tabular}{|c|c|c|c|c|c|c|c|c|c|c|c|c|c|c|c|c|}
\hline \multirow{5}{*}{ 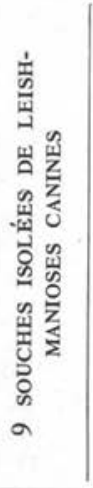 } & \multirow{5}{*}{ 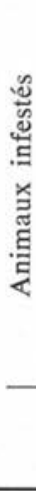 } & 80 & $\begin{array}{l}\stackrel{7}{\mathcal{f}} \\
\stackrel{2}{7}\end{array}$ & & $\therefore$ & $a^{\circ}$ & $\begin{array}{l}8 \\
0\end{array}$ & $\begin{array}{l}\stackrel{2}{2} \\
\text { ñ }\end{array}$ & . & . & . & . & . & & $\therefore$ & . \\
\hline & & 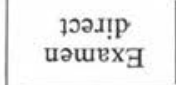 & $\infty$ & . & 0 & 0 & 0 & $\nabla$ & . & . & . & . & $\cdot$ & . & 0 & . \\
\hline & & $\therefore$ & $\therefore$ & & 2 & $\mid \begin{array}{l}0 \\
0\end{array}$ & $\begin{array}{l}30 \\
0\end{array}$ & $\begin{array}{l}\therefore 0 \\
\text { in }\end{array}$ & · & . & . & . & . & & 5 & . \\
\hline & & $\begin{array}{l}\text { NNN Ins } \\
\text { ว.มn][nว }\end{array}$ & $\stackrel{\infty}{\sim}$ & . & - & 0 & - & 0 & . & . & . & . & . & & 0 & . \\
\hline & & 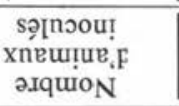 & $\stackrel{\infty}{\infty}$ & . & + & N & 오 & $=$ & . & . & . & . & $\cdot$ & . & $\nabla$ & . \\
\hline 起䍃 & & $\therefore$ & $\begin{array}{c}4 \\
80\end{array}$ & - & . & $\begin{array}{l}8 \\
0\end{array}$ & $\begin{array}{l}80 \\
6 \\
0\end{array}$ & . & $\theta^{\circ}$ & . & . & . & . & . & - & . \\
\hline 可 & 节 & 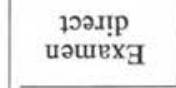 & $m$ & - & - & 0 & $\mathrm{~N}$ & . & 0 & - & . & . & . & . & - & . \\
\hline 氙: & 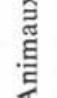 & 80 & so & . & . & $\begin{array}{l}20 \\
0\end{array}$ & $\begin{array}{l}80 \\
2\end{array}$ & . & $\therefore$ & . & . & . & . & . & . & $\cdot$ \\
\hline 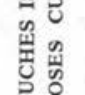 & & $\begin{array}{l}\text { NNN Ins } \\
\text { ว.m![nJ }\end{array}$ & $n$ & - & - & 0 & $m$ & . & 0 & . & . & . & $\cdot$ & . & . & . \\
\hline $\begin{array}{l}\delta_{n} \bar{z} \\
\dot{q}\end{array}$ & & 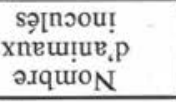 & $F$ & - & . & r & $\approx$ & . & N & . & . & . & $\cdot$ & . & . & . \\
\hline 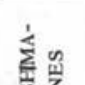 & & $\therefore$ & $\therefore$ & $\therefore$ & . & $\begin{array}{l}8^{\circ} \\
0\end{array}$ & \&̊ & $\stackrel{\circ}{2}$ & 20 & $8^{\circ}$ & so & 80 & $\begin{array}{l}8 \\
0\end{array}$ & $\begin{array}{l}0 \\
0\end{array}$ & $\begin{array}{l}50 \\
0\end{array}$ & $\begin{array}{l}80 \\
0\end{array}$ \\
\hline 要 & 总 & 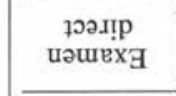 & 0 & 0 & . & 0 & $\infty$ & $N$ & 0 & 0 & 0 & 0 & 0 & 0 & 0 & 0 \\
\hline 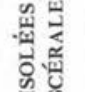 & 袢 & $\delta^{\circ}$ & $\begin{array}{l}\stackrel{8}{2} \\
\simeq\end{array}$ & or & & $\begin{array}{l}80 \\
0\end{array}$ & $\therefore$ & $\therefore$ & $\begin{array}{l}20 \\
0\end{array}$ & $\therefore$ & so & $\begin{array}{l}\circ \\
0\end{array}$ & $\begin{array}{l}30 \\
0\end{array}$ & $\begin{array}{l}8 \\
0\end{array}$ & $\therefore$ & $\begin{array}{l}8 \\
0\end{array}$ \\
\hline 证 & & $\begin{array}{l}\text { NNN Ins } \\
\text { ə.มn\}[n }\end{array}$ & $\vec{\sim}$ & 0 & - & 0 & $\approx$ & $N$ & 0 & 0 & 0 & 0 & 0 & 0 & 0 & 0 \\
\hline $\begin{array}{l}0 \\
\text { in } \\
\text { in }\end{array}$ & & 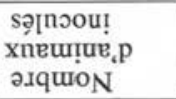 & ले & $\varrho$ & . & N & 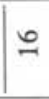 & 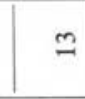 & 6 & $r$ & in & $N$ & $N$ & - & - & $m$ \\
\hline & & & 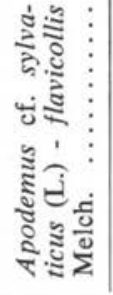 & 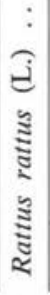 & 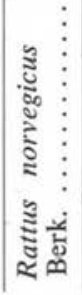 & 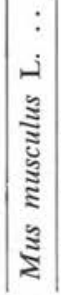 & 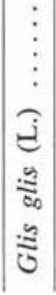 & 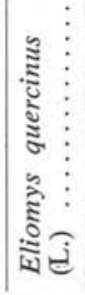 & 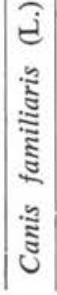 & 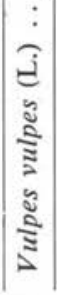 & 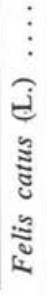 & 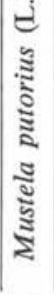 & 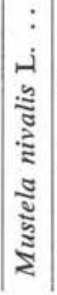 & 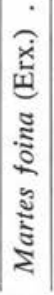 & 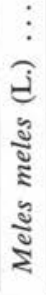 & 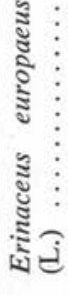 \\
\hline
\end{tabular}


l'hémoculture. La fixation du complément était positive au $1 / 80^{\circ}$ chez les deux animaux inoculés. Le témoin restait négatif.

\section{$3^{\circ}$ Coaptation trophique « vecteur-réservoir ».}

Mentionnons enfin, qu'une étude des préférences alimentaires du « vecteur habituel », Phlebotomus ariasi, a pu être menée sur le Renard comparativement au Chien, aux Rongeurs et aux Reptiles. Pour ce faire, l'animal, placé sous une moustiquaire bordée, restait en contact, pendant une nuit, avec un certain nombre de Phlébotomes non gorgés. Le matin, les femelles étaient recapturées et identifiées. Les résultats définitifs étaient exprimés en $\%$ de $q$ gorgées (tabl. 2).

TABLEAU II

\begin{tabular}{|c|c|c|c|}
\hline EsPÈCES & q gorgées & q non gorgées & $\%$ \\
\hline Chien (Canis familiaris) ..... & 243 & 24 & $91 \%$ \\
\hline Renard (Vulpes vulpes) ...... & 149 & 123 & $55 \%$ \\
\hline Lapin (Oryctolagus cuniculus). & 72 & 201 & $26 \%$ \\
\hline Lérot (Eliomys quercinus) ... & 0 & 185 & $0 \%$ \\
\hline Mulot (Apodemus sylvaticus). & 0 & 75 & $0 \%$ \\
\hline Lézard vert (Lacerta viridis). & 0 & 218 & $0 \%$ \\
\hline $\begin{array}{l}\text { Couleuvre d'Esculape (Coluber } \\
\text { longissimus) } \ldots \ldots \ldots \ldots \ldots \ldots\end{array}$ & 0 & 307 & $0 \%$ \\
\hline
\end{tabular}

Par cette méthode, qui confirme la grande appétence de Phlebotomus ariasi pour le Chien $(91 \%)$, on constate que le Renard est exploité de manière non négligeable (55\%) à l'inverse des Rongeurs (Lapin, $26 \%$; Lérot, Loir et Mulot, $0 \%$ ) et des Reptiles (Lézard vert et Couleuvre d'Esculape, $0 \%$ ).

Ainsi, la découverte princeps de l'infestation leishmanienne naturelle du Renard, la possibilité de reproduire expérimentalement la maladie chez cet animal, constituent autant d'observations du plus haut intérêt, tant théorique que pratique. A l'avenir, il devrait en être tenu compte dans toute enquête épidémiologique menée en pays méditerranéens où d'autres Canidés sauvages, tels le Chacal ou le Fennec (Fennec zerda L.) peuvent d'ailleurs intervenir. Précisons toutefois, qu'il serait prématuré de définir sur le vu de ces seules données la place exacte des réservoirs selvatiques dans le complexe leishmanien. Interviennent-ils dans un «mode habituel » ou sont-ils atteints accidentellement à partir d'un réservoir canin? Servent-ils d' « hôtes de liaison » ou évoluent-ils pour leur propre 
compte à l'intérieur d'un cycle semi-autonome? Autant de questions dont les réponses ne sauraient être valablement apportées sans une nouvelle enquête écologique (1).

\section{Bibliographie}

AdLer (S.), 1964. - Leishmania. Advances in Parasitology. Academic Press éd. New-York, 2: $35-91$.

Adler (S.) et THEodor (O.), 1931. - Investigations on Mediterranean Kala-Azar. III. The sandflies of the Mediterranean Basin. Distribution and bionomics of sandflies in Catania and District. Proc. Roy. Soc. London, 108: 464-480.

Ansari (N.), 1962. - The Leishmanioses as a world problem. Sc. Rep. Ist. Super. Sanita, $2: 63-67$.

Biocca (E.) et CorradetTi (A.), 1952. - Babesia missirolii n. sp., parassita del tasso (Meles taxus). (English summary). Riv. Parassit., 13: 17-19.

Colas-Belcourt (J.), 1956. - Phlébotomes et Leishmanioses autochtones en France. Proc. Tenth. Int. Congr. Ent., $3: 825-828$.

Corradetti (A.), 1964. - Mediterranean Kala-Azar. Parass., 6: 275-277.

DeANE (L. M.), 1958. - Epidemiologia e profilaxia do calazar americano. Rev. Bras. Malar. Doen. Trop., 10: 431-450.

DeAne (L.) et DeAne (M. P.), 1955. - Observacoes preliminares sobre importancia comparativa do homem, do cao e da raposa (Lycalopex vetulus) como reservatorios da Leishmania donovani em area endemica de Calazar, no Ceara. O Hospital, 48 : 61-70.

Deane (M.) et Deane (L. M.), 1954. - Infeccao experimental do Phlebotomus longipalpis em raposa Lycalopex vetulus naturalmente parasitada pela Leishmania donovani. O Hospital, 46 : 651-653.

Fraga de Azevedo (J.), 1948. - Sur la transmission du Kala-Azar méditerranéen. Anais Inst. Med. Trop., $5: 269-286$.

Garnham (P.), 1965. - The Leishmanias, with special reference to the role of animal reservoirs. Am. Zool., 5 : 141-151.

Hourn (R.), 1963. - Données épidémiologiques et déductions prophylactiques sur les Leishmanioses autochtones en France. Ann. Paras. Hum. Comp., $38: 379-438$.

KIRK (R.), 1956. - Studies in leishmaniasis in the Anglo-Egyptian Sudan. XII. Attempts to find the reservoir host. Trans. Roy. Soc. trop. Med. Hyg., 50: 169-177.

Latyshev (N. I.), Kryukova (A. P.) et Povalishina (T. P.), 1951. - Essays on the regional parasitology of Middle Asia. I. Leishmaniases in Tajikistan. Materials for the medical geography of Tadjik SSR. (Results of expeditions in 1945-47). Problems of Regional, General and Experimental Parasit. Med. Zool., Moscow, 7: 35-62.

Nicolle (C.), 1908. - Nouvelles acquisitions sur le Kala-azar; cultures, inoculation au chien, étiologie. C.R. Acad. Sc., 146: 498-499.

(1) Ainsi, pour ne citer qu'un exemple élémentaire, la simple densité du Renard au kilomètre carré, est totalement inconnue dans le « Midi » de la France. 
PARROT (L.), 1934. - La transmission naturelle des Leishmanioses du bassin méditerranéen. Bull. Organ. Hyg. S.D.V., $3: 210-228$.

Pavlovsky (E. N.), 1927. - Histoire de la découverte du parasite du Bouton d'Orient. Pensee Med. d'Uzbekistane, 2 : 16-19 (en russe).

PetrishcheVA (P. A.), 1935. - The fauna, biology and ecology of the sandflies of Turkmenistan. In : Parasites, Carriers and Venomous animals, Moscou, Leningrad, 198-202.

Rioux (J.-A.), Albaret (J.-L.), Bres (A.) et Dumas (A.), 1966. - Babesia missirolii, Biocca et Corradetti 1952 chez le Blaireau [Meles meles (L.)] du Sud de la France. Journ. of Protozool., 13, 3, suppl., p. 41.

SYMmers (W.S.), 1960. - Transmission leishmaniasis acquired by contagion; a case of marital infection in Britain. Lancet, $1: 127-132$.

VLasov (U. P.), 1932. - On the finding of sandflies in the environs of Szchkabad in the burrows of rodents Rhombomys opimus, Licht. and Spermophilopsis leptodactylus Licht. Mag. Paras. Inst. Zool. Acad. Sc. U.S.S., 3: 89-102. 